\title{
Effects of Near Infrared light Application Combined with Aerobic Exercise on Excessive Abdominal Fat and Obesity
}

\author{
Asif $\mathrm{Ali}^{1 *}$, Kwangmin $\mathrm{Ryu}^{1}$, Sungwoon $\mathrm{Kim}^{1}$ and $\mathrm{Ren} \mathrm{Xi} \mathrm{Li}^{2}$ \\ ${ }^{1}$ Department of Physical Education, Kyungpook National University, \\ Daegu, South Korea \\ ${ }^{2}$ Depratment of Sport Psychology, University of Petroleum, China \\ *lyu42@daum.net
}

\begin{abstract}
This study was conducted to examine the effects of a near infrared-light-emitting diode (NIR-LED) in conjunction with low endurance exercise on obesity and abdominal fat. Twenty-six overweight adolescents aged 14-15 years were divided into an experimental and control group. Participants in the experimental group performed endurance exercise on a treadmill while wearing NIR-LED belts on their abdomens. The exercise intensity, duration, and frequency were $50 \%$ VO2 max, $45 \mathrm{~min} / \mathrm{session}$ and three days per week for four weeks. Participants in the control group underwent the same exercise protocol as those in the experimental group. The only difference between both groups was that the participants in the control group used belts that have no NIR-LED. The results of this experiment demonstrated that participants in the experimental group showed a significantly reduced body mass index (BMI), circumference of waists, and percentage of body fat when compared to participants in the control group. These results suggest that application of the NIR-LED is an effective method for the treatment of obesity and reducing abdominal fat. We postulated that NIR stimulated the biological functions of cells situated at the abdominal region, which made more fat available for burning during endurance exercise. In addition, NIR-LED and exercise increased body temperature during treatment, which further enhanced mobilization of fatty tissues. These underlying mechanisms support the view that both NIR and low intensity exercise show synergistic effects for reducing fat. However, further research is needed to validate the results presented herein.
\end{abstract}

Keywords: Obesity, Abdominal fat, NIR-LED, Endurance exercise, Light therapy mechanisms

\section{Introduction}

The prevalence of obesity in the general population and particularly in children has become an epidemic issue and an unusual health concern across the world [1]. The numbers of obese and overweight members of the population are consistently increasing at an alarming rate in developed and developing countries [2]. Various pathological [3] socioeconomic [4] psychological [5] and health problems are linked to excessive fat [6]. Research has demonstrated that several mental disorders and a decline in cognitive functions also associated with obesity and overweight [7,8].These harmful consequences can result in a low quality of life [9]. It is well known that excessive body fat has dangerous health consequences; however, abdominal obesity appears to be particularly detrimental to human health. Specifically, this type of obesity is strongly connected to type 2 diabetes, heart diseases, Alzheimer's disease, hypertension, and metabolic syndrome [10]. Furthermore, there is a strong correlation between increased abdominal fat and increased death risk [11]. Therefore, reductions in belly fat and overall obesity have generally been the focus of health research.

Several interventions and treatments associated with surgery, nutrition, medication and exercise have been prescribed to treat obesity and overweight [12-16]. However, more advanced and powerful techniques are required to overcome subcutaneous fat located around the abdomen and stomach region. Here, we introduce a novel and scientific approach to address this issue; namely, the application of NIR (near infrared) LED (light-emitting diode) belts to the bellies of overweight participants, combined with low intensity aerobic exercise on a treadmill. The use of the NIR (near 
infrared)-LED (light-emitting diode) at certain wavelengths (630 to $1000 \mathrm{~nm}$ ), intensities, frequencies and doses has been shown to be potentially beneficial to wound healing processes in several clinical studies[17, 18]. Specifically, it appears to be beneficial for nerve regeneration, peripheral nerve repair, treatment of dermatitis, optical nerve degeneration, prevention of oral mucositis, and healing of venous ulcers and retinal toxicity [19-21]. Moreover, NIR-LED has been shown to have protective effects against neural damage and Parkinson's disease [22, 23].

The beneficial effects of NIR-LED in clinical and therapeutic settings are associated with its strong bio-stimulatory effects on biological tissues, which cause increased metabolism. However, a slow metabolism is attributed to fat, and NIR-LED is supposed to stimulate fat metabolism, which may lead to reduced abdominal fat. Previous studies have shown that NIR-LED radiation works under thermal and biochemical mechanisms to reduce fat beneath the skin. First, NIR radiation penetrates deeper under the skin, targets fat tissues, and increases temperature, which results in liquefied fat tissues and facilitates fat metabolism [24]. Second, NIR-LED radiation is associated with excitation of cytochrome coxidase (CCO) and complex IV enzyme activity, which results in accelerated mitochondrial energy metabolism[25]. In addition to NIR-LED, the fuel source of energy metabolism during aerobic exercise is heavily fat dependent. Due to utilization of the same energy mechanisms, the combined use of NIR-LED radiation and aerobic exercise may show synergic effects in reducing local body fat in overweight subjects. Past research has suggested that combining aerobic exercise with other weight-losing variables has resulted in synergistic effects [14]. Therefore, this study was conducted to determine if application of a NIR-LED device along with aerobic exercise had synergistic effects on the reduction of local body fat.

\section{Methods}

\subsection{Participants}

Twenty-six overweight but otherwise healthy male students from a local school with ages, body mass index (BMI), and body weights ranging from 14-15 years (mean, 14.8), 25.1-32.1 (mean, 28.61), and 70.1-89.6 (mean, 78.6), respectively, were recruited to participate in this study. We divided the participants into an experimental group and a control group. Informed consent was obtained from both the parents and participants, approved by our Institutional Review Board. Additionally, all participants answered a questionnaire regarding their health status and medication. Participants with symptoms from severe illnesses or were taking medication that could affect their exercise performance on the treadmill were excluded from the experiment during the pre-screening procedure. Additionally, the participants were free to leave the experiment whenever they wanted. Each participant received 30 US dollars as compensation.

\subsection{Procedure and Apparatus}

Upon arriving at the laboratory, all the participants were introduced about the experimental procedure, and apparatus (e.g., Treadmill machine, wearing the belts, controlling exercise intensity). They were further explained about safety measures regarding use of apparatus and exercise. They were informed that in case of arising any kind of health problem, pain or symptoms of dizziness, they should stop exercise and report to the assistant investigator. Prior to the experiment, we administered a VO2 max test to determine the percentage of VO2 max for each participant in order to control the $50 \%$ exercise intensity condition. Participants in the experimental group followed an aerobic exercise protocol on treadmill machines while also using NIR-LED belts (NIR-7 Healthcare, Korea) with wavelengths of 700-960 nm on their abdomens. For the purpose of ensuring experimental conditions and safety measures, three assistant investigators were present during the whole intervention period. The exercise protocol was comprised of a 45 min walk with 50\% VO2 max, three days a week for four weeks. Participants in the control group followed the same exercise protocol, but wore belts that had no NIR-LED. 


\subsection{Dependent Variables}

Following the VO2 max test, participants in both groups underwent testing in accordance with the pre and post experimental design. The testing protocol consisted of measurement of 1 ) the sum of the circumferences of the abdomen, 2) body mass index (BMI), and 3) percentage of body fat.

\subsection{Data Analysis}

A 2 (groups: exercise with NIR-LED and exercise only) x 2 (tests: pre- and post) ANOVA with repeated measures was conducted to examine the effects on the sum of circumferences of the abdomen, body mass index (BMI), and body fat $\%$. The alpha level was set to 0.05 .

\section{Results}

\subsection{Body Mass Index (BMI)}

As shown in Figure 1, analysis of the BMI revealed that there was no significant main effect for Group $F(1,24)=0.523, p>0.05, \eta^{2}=0.021$. However, there were significant effects for Time $F(1$, $24)=83.32, p<0.01, \eta^{2}=0.776$ and Group by Time interaction $F(1,24)=10.85, p<0.01, \eta^{2}=$ $0.723)$.

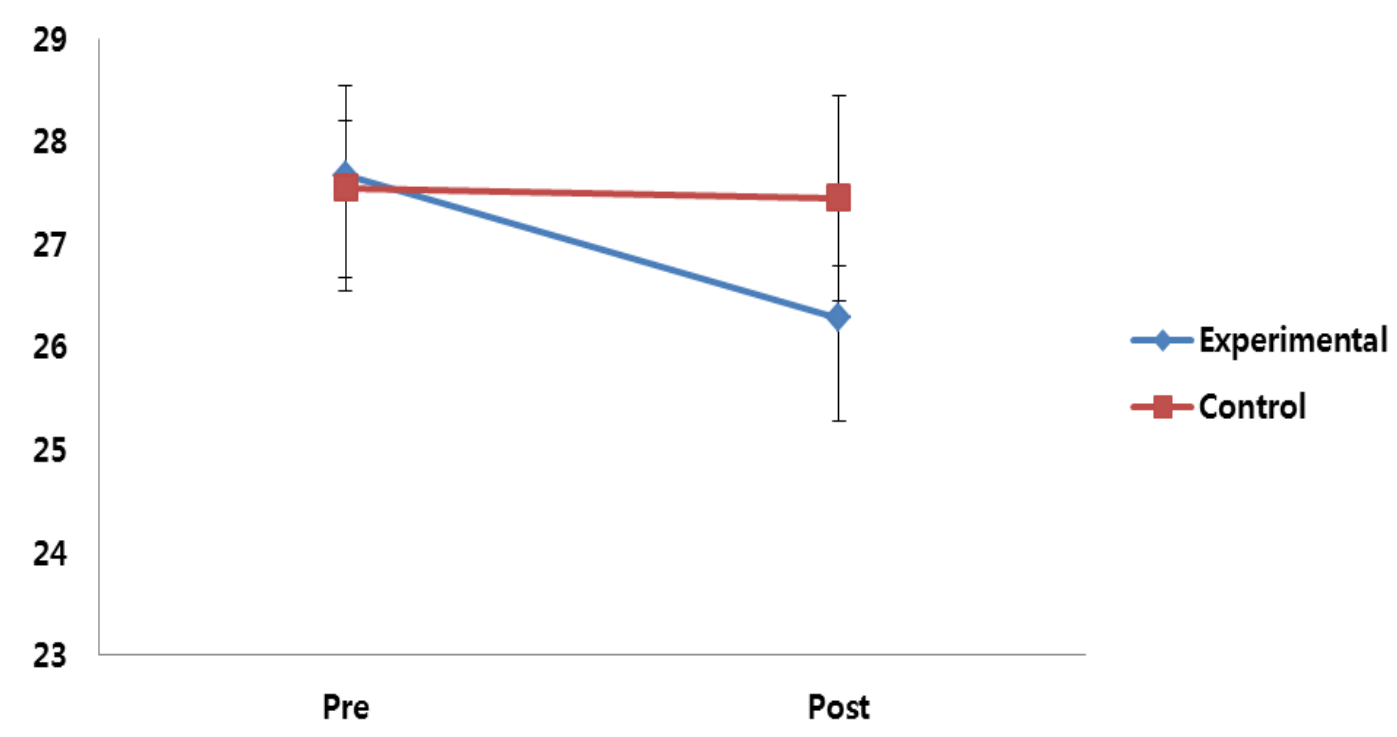

Figure 1. Reduction in Body Mass Index (BMI) as a result of application of NIR-LED belts on the bellies of the partcipants in the experimental group during 4 weeks of excercise

\subsection{Abdominal Circumference}

Analysis of the waist circumference indicated that the main effect for Group $F(\mathrm{I}, 24)=1.293, P>$ $0.05, \eta^{2}=0.051$, was not significant. However, the main effect for Time $F(1,24)=233.862, P<.000$, $\eta^{2}=.907$, and Group by Time interaction $F(1,24)=127.723, P<.000, \eta^{2}=.842$ were significant (see Figure 2). 


\section{Abdominal Cercumference}

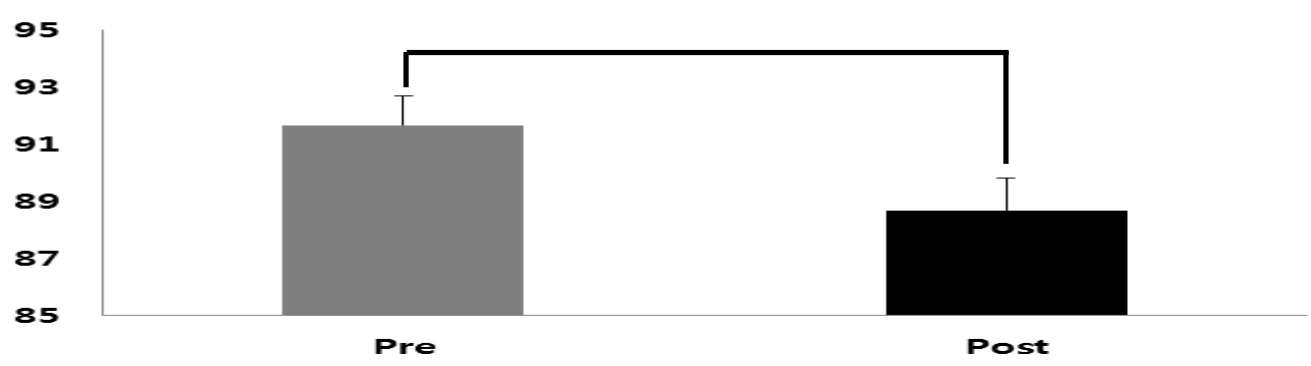

Figure 2. Reduction in the Circumference of Waists Due to use of NIR-LED Belts on the Bellies of Participants during Four Weeks of Excercise

\subsection{Percentages of Body Fat}

As shown in Figure 3, analysis of the percentage of body fat revealed a significant main effect for Group $F(1,24)=14.601, P<0.001, \eta^{2}=.378$, Time $F(1,24)=41.268, P<0.001, \eta^{2}=0.632$, and Group by Time interaction $F(1,24)=44.018, P<0.000, \eta^{2}=0.647$.

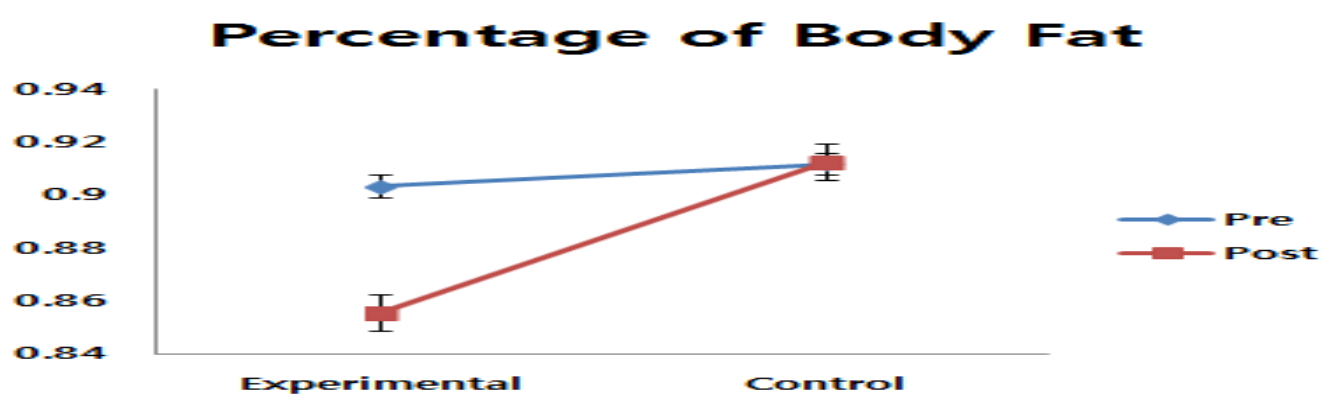

Figure 3. Decrease in Body Fat in Participants who used NIR-LED Belts when Compared to those who Performed Exercise alone for 4 Weeks

\section{Discussion}

This study was conducted to assess the effectiveness of use of NIR-LED in conjunction with low endurance exercise based on the reduction of abdominal fat and weight loss in overweight adolescents. The main findings of this study indicated that members of the experimental group showed remarkably decreased fat when compared with those in the control group as indicated by a significant reduction in BMI, circumference of the waist, and percentage of body fat. These findings suggest that the application of a NIR-LED belt offers unique beneficial effects for the treatment of obesity and an immensely useful tool for burning abdominal fat. Specifically, these outcomes provide evidence of a synergistic effect between NIR-LED and low intensity endurance exercise in association with augmented metabolism of fatty tissues.

The results demonstrate that participants who used the NIR-LED belt in conjunction with exercise showed a decrease in BMI of 5.02\% when compared with those who performed only aerobic exercise during the experimental period. These findings suggest that NIR-LED combined with aerobic exercise has beneficial effects for weight loss. This effect might be due to the fact that cytochrome $\mathrm{C}$ oxidase in the mitochondria absorbs the light $[24,26]$, which leads to an accelerated mitochondrial energy production mechanism [27], resulting in more energy becoming available to exercise the body, and therefore increased burning of calories and a reduction in body weight. These results are consistent with those of a previous study that indicated body mass index and body weight decreased significantly in female obese participants who used water-filtered infrared-A (wIRA) while performing endurance exercise on a bicycle ergometer for 4 weeks[28]. In that study, the major body parts including the thigh, hip, and abdomen were irradiated by water-filtered infrared-A (wIRA) lamps during ergometer 
exercise, which produced heat, making it difficult to use in hot weather. In contrast, we used NIRLED belts that have a selective effect on abdominal fat and could therefore be used in all weather conditions to reduce weight.

The results of the circumference of the waist revealed a greater reduction in abdominal fat for the NIR-LED condition $(5.65 \%)$ than the control condition $(0.84 \%)$. In addition, the NIR-LED condition yielded a greater decrease in the percentage of body fat than those of the control condition. A 5.55\% reduction in body fat percentage was observed in the NIR-LED participants; however, no decrease in body fat was observed in the control group.

The significant reduction in abdominal fat and overall body fat in response to the application of NIR-LED on the participants' bellies while exercising observed in this study is similar to the results of previous studies related to lipolysis [28, 29]. Several factors may explain these benefits of using NIR-LED belts. Specifically, NIR may have biochemical and thermal effects on biological tissues. Research has demonstrated that NIR light penetrates the abdominal skin deeply into adipose tissue located under the skin $[30,31]$, where photoreceptor molecules that absorb the light, such as myoglobin, cytochrome C oxidase, and hemoglobin, are located [32] Karu (1999) illustrated that NIR light elevated the functional activity of cytochrome $\mathrm{C}$ oxidase, which is associated with promoting oxidative metabolism in the mitochondria and leads to increased ATP production, resulting in further energy becoming available for the metabolism of fatty tissues[25, 33 ]. Previous studies have shown that NIR light irradiation promotes nerve regeneration by enhancing oxidative processes of the mitochondria in vivo [19]. Several studies have also reported bio-stimulatory effects of NIR light on wound healing [17], cell growth [34], bone repair and reducing inflammation [35], as well as on the healing of nipple trauma in breastfeeding women [36]. Additionally, Anja Heselich et al., (2011) indicated that the principle mechanism of positive [36] consequences of NIR devices primarily originates from its potential to improve cellular metabolism [37]. Based on the present results and those of previous studies, we postulate that NIR-LED stimulated the metabolism of adipose tissues, resulting in belly fat becoming a source of ATP production, which was consumed by the aerobic exercise. These assumptions are supported by the results of other studies that showed that functional alterations associated with NIR-LED lead to a delay in fatigue during exercise and increases in exercise tolerance on a treadmill $[38,39]$. The findings from those studies clearly demonstrate that NIR-LED primarily serves to induce biochemical reactions leading to energy production in humans.

Taking together, these findings correspond to the results of studies that demonstrated the positive effects of a combination of light therapy and exercise on the reduction of weight and abdominal fat [40]. A previous study indicated that water-filtered infrared-A (wIRA) irradiation in conjunction with aerobic exercise could potentially reduce local body fat and weight in obese individuals [28]. Although the results of our study showed clear beneficial effects of NIR-LED for abdominal fat reduction and overall body weight loss, more studies are needed to validate these findings on other body parts and in combination with different types of exercise using participants of different ages, genders, and levels of obesity. It will be more interesting to assess the effects of NIR-LED based on a combination of nutrition interventions along with multiple light therapies including water-filtered infrared-A (wIRA) and low-level laser therapy (LLLT).

Overall, the application of NIR-light-emitting diode (LED) therapy offers an innovative, noninvasive, easy to use, safe, and promising method for controlling obesity and abdominal fat.

\section{Abbreviations}

NIR, near infrared; LED, light-emitting diode; BMI, body mass index; ATP, adenosine triphosphate; wIRA, water-filtered infrared-A; LLLT, low-level laser therapy

\section{References}

[1] J. E. Shaw, R. A. Sicree and P. Z. Zimmet, "Global estimates of the prevalence of diabetes for 2010 and 2030", Diabetes Research and Clinical Practice, vol. 87, no. 1, (2010), pp. 4-14.

[2] P. Hossain, B. Kawar and M. E. 1. Nahas, "Obesity and Diabetes in the Developing World-A Growing Challenge", The new England Journal of Medicine ,vol. 356, no. 3, (2007), pp. 213-215.

[3] F. X. Pi-Sunyer, "The Obesity Epidemic: Pathophysiology and Consequences of Obesity", vol. 10, no. 12, (2002), pp. 97-104. 
[4] D. Yach, D. Stuckler and K. D. Brownell, "Epidemiologic and economic consequences of the global epidemics of obesity and diabetes", Nature Medicine, vol. 12, (2006), pp. 62-66.

[5] G. Zhao, C. Li, E. S. Ford, J. Tsai, S. T. Dhingra, J. B. Croft, L. R. McKnight-Eily and L. S. Balluz, "Associations between Overall and Abdominal Obesity and Suicidal Ideation among US Adult Women”, Journal of Obesity, (2012), pp. 9.

[6] V. J. Lawrence and P. G. Kopelman, "Medical consequences of obesity. Clinics in Dermatology", vol. 22, no. 4, (2004), pp. 296-302.

[7] M. E. Tascilar, D. Turkkahraman, O. Oz, M. Yucel, M. Taskesen, I. Eker, A. Abaci, R. Dundaroz and U. H. Ulas, "P300 auditory event-related potentials in children with obesity: is childhood obesity related to impairment in cognitive functions?", Pediatric Diabetes, vol. 12, no. 7, (2011), pp. 589-595.

[8] A. A. Mather, B. J. Cox, M. W. Enns and J. Sareen, "Associations of obesity with psychiatric disorders and suicidal behaviors in a nationally representative sample", Journal of Psychosomatic Research, vol. 66, no. 4, (2009), pp. 277285.

[9] K. C. Swallen, E. N. Reither, S. A. Haas and A. M. Meier, "Overweight, Obesity, and Health-Related Quality of Life Among Adolescents: The National Longitudinal Study of Adolescent Health”, Pediatrics, vol. 115, no. 2, (2005), pp. $340-347$.

[10] A. A. Mahabadi, J. M. Massaro, G. A. Rosito, D. Levy, J. M. Murabito, P. A. Wolf, C. J. O'Donnell, C. S. Fox and U. Hoffmann, "Association of pericardial fat, intrathoracic fat, and visceral abdominal fat with cardiovascular disease burden: the Framingham Heart Study", European Heart Journal, vol. 30, no. 7, (2009), pp. 850-856.

[11] X. Zhang, X. Shu, G. Yang, H. Li, H. Cai, Y. Gao and W. Zheng, "Abdominal Adiposity and Mortality in Chinese Women", Archive of Internal Medecine, vol. 167, no. 9, (2007), pp. 886-892.

[12] C. M. Caperchione, C. Vandelanotte, G. S. Kolt, M. Duncan, M. Ellison, E. George and W. K. Mummery, "What a Man Wants: Understanding the Challenges and Motivations to Physical Activity Participation and Healthy Eating in Middle-Aged Australian Men”, American journal of Mens Health, vol. 6, no. 6, (2012), pp. 453-461.

[13] V. E. P. P. Lemmens, A. Oenema, K. I. Klepp, B. H. Henriksen and J. Brug, "A systematic review of the evidence regarding efficacy of obesity prevention interventions among adults", Obesity Reviews, vol. 9, no. 5, (2008), pp. 446455.

[14] A. M. Hill, J. D. Buckley, K. J. Murphy and P. R. C. Howe, "Combining fish-oil supplements with regular aerobic exercise improves body composition and cardiovascular disease risk factors", American Journal of Clinical Nutrition, vol. 85, no. 5, (2007), pp. 1267-1274.

[15] J. Sabaet and M. Wien, "Vegetarian diets and childhood obesity prevention", American Journal of Clinical Nutrition, vol. 91, no. 5, (2010), pp. 1525-1529.

[16] G. J. Heijden, Z. J Wang, Z. D. Chu, P. J. J. Sauer, M. W. Haymond, L. M. Rodriguez and A. L. Sunehag, "A 12-Week Aerobic Exercise Program Reduces Hepatic Fat Accumulation and Insulin Resistance in Obese, Hispanic Adolescents", Obesity, vol. 18, no. 2, (2010), pp. 384-390.

[17] H. T. Whelan, R. L. Smits Jr, E. V. Buchman, N. T. Whelan, S. G. Turner, D. A. Margolis, V. Cevenini, H. Stinson, R. Ignatius, T. Martin, J. Cwiklinski, A. F. Philippi, W. R. Graf, B. Hodgson, L. Gould, M. Kane, G. Chen and J. Caviness, "Effect of NASA light-emitting diode irradiation on wound healing", Journal of Clinical Laser Medicine \& Surgery, vol. 19, no. 6, (2001), pp. 305-314.

[18] M. D. Skopin and S. C. Molitor, "Effects of near-infrared laser exposure in a cellular model of wound healing", Photodermatology, Photoimmunology \& Photomedicine, vol. 25, no. 2, (2009), pp. 75-80.

[19] M. Ishiguro, K. Ikeda and K. Tomita, "Effect of near-infrared light-emitting diodes on nerve regeneration", Journal of Orthopaedic Science, vol. 15, no. 2, (2010), pp. 233-239.

[20] M. Ishiguro, K. Ikeda, H. Noriyuki, K. Tada and K. Tomita, "The effect of near-infrared light-emitting diodes on the anti oxidation of nerve regeneration chamber fluid", Journal of Orthopaedic Science, vol. 15, no. 2, (2008), pp. 233239.

[21] K. S. Caetano, M. A. C. Frade, D. G. M. Minatel, L.A. Santana and C. S. Enwemeka, "Phototherapy Improves Healing of Chronic Venous Ulcers", Photomedicine and Laser Surgery, vol. 27, no. 1, (2009), pp. 111-118.

[22] J. C. Rojas, J. Lee, J. M. John and F. Gonzalez-Lima1, "Neuroprotective Effects of Near-Infrared Light in an In Vivo Model of Mitochondrial Optic Neuropathy", The Journal of Neuroscience, vol. 28, no. 50, (2008), pp. 13511-13521.

[23] B. J. Quirk, K. D. Desmet, M. Henry, E. Buchmann, M. Wong-Riley, J. T. Eells and H. T. Whelan, "Therapeutic effect of near infrared (NIR) light on Parkinson's disease models", Frontiers in Bioscience (Elite Ed). vol. 1, no. 4, (2011), pp. 818-23.

[24] I. Y. Yanina, V. V. Tuchin, N. A. Navolokin, O. V. Matveeva, A. B. Bucharskaya and G. N. Maslyakova, "Fat tissue histological study at NIR laser treatment of the skin in vivo", European Conference on Biomedical Optics, Munich, Germany, (2011), pp. 809215-809215

[25] T. Karu, "Primary and secondary mechanisms of action of visible to near-IR radiation on cells", Journal of Photochemistry and Photobiology B: Biology, vol. 49, no. 1, (1999), pp. 1-17.

[26] T. I. Karu, "Multiple roles of cytochrome c oxidase in mammalian cells under action of red and IR-A radiation", IUBMB Life, vol. 62, no. 8, (2010), pp. 607-610.

[27] J. T. Eells' M. T. T. Wong-Riley, J. VerHoeve, M. Henry, E. V. Buchman, M. P. Kane, L. J. Gould, R. Das, M. Jett, B. D. Hodgson, M. David and H. T. Whelan, "Mitochondrial signal transduction in accelerated wound and retinal healing by near-infrared light therapy", Mitochondrion, vol. 4, no. 5-6, (2004), pp. 559-567.

[28] M. Frank, H. Gerd, O. Roy, D. Wolfgang, and S. Gerd, "Influence of water-filtered infrared-A (wIRA) on reduction of local fat and body weight by physical exercise", German Medical Science, vol. 4, (2006). 
[29] S. R. Mordon, B. Wassmer, J. P. Reynaud and J. Zemmouri, "Mathematical modeling of laser lipolysis", BioMedical Engineering OnLine, vol. 7, no. 1, (2008), pp. 1-13.

[30] Z. Zhang, B. Wang, Q. Nie, Q. Luo and H. Gong, "Portable muscle oxygenation monitor based on near infrared spectroscopy", Frontiers of Optoelectronics in China, vol. 2, no. 3, (2009), pp. 248-252.

[31] S. E. Krite, P. Wollmer, S. Andersson-Engels and J. Akeson, "Physiological influence of basic perturbations assessed by non-invasive optical techniques in humans", Applied Physiology, Nutrition, and Metabolism, vol. 36, no. 6, (2011), pp. 946-957.

[32] D. M. Mancini, L. Bolinger, H. Li, K. Kendrick, B. Chance and J. R. Wilson, "Validation of near-infrared spectroscopy in humans", Journal of Applied Physiology, vol. 77, no. 6, (1994), pp. 2740-2747.

[33] M. R. Hamblin and T. N. Demidova, "Mechanisms of low level light therapy", Paper presented at the Biomedica Optics, (2006).

[34] M. T. Pagin, F. A. de Oliveira, A. C. P. Sant'Ana, M. L. R. de Rezende, S. L. A. Greghi and C. A. Damante, "Laser and light-emitting diode effects on pre-osteoblast growth and differentiation", Lasers in Medical Science, (2012).

[35] A. B. Pinheiro, L. G. P. Soares, A. F. S. Barbosa, L. M. P. Ramalho and J. N. dos Santos, "Does LED phototherapy influence the repair of bone defects grafted with MTA, bone morphogenetic proteins, and guided bone regeneration? A description of the repair process on rodents", Lasers in Medical Science, vol. 27, no. 5, (2012), pp. 1013-1024.

[36] M. E. A. Chaves, A. R. Araujo, S. F. SAntos, M. Pinotti and L. S. Oliveira, "LED Phototherapy Improves Healing of Nipple Trauma: A Pilot Study”, Photomedicine and Laser Surgery, vol. 30, no. 3, (2012), pp. 172-178.

[37] A. Heselich, F. Frohns, A. Frohns, S. C. Naumann and P. G. Layer, "Near-Infrared Exposure Changes Cellular Responses to Ionizing Radiation", Photochemistry and Photobiology, vol. 88, no. 1, (2012), pp. 135-146.

[38] F. R. Paolillo, J. C. Milan, I. V. Aniceto, S. G. Barreto, J. R. Rebelatto, A. Borghi-Silva, N. A. Parizotto, C. Kurachi and V. S. Bagnato, "Effects of Infrared-LED Illumination Applied During High-Intensity Treadmill Training in Postmenopausal Women", Photomedicine and Laser Surgery, vol. 29, no. 9, (2011), pp. 639-645.

[39] F. R. Paolillo, A. V. Corazza, A. Borghi-Silva, N. A. Parizotto, C. Kurachi and V. S. Bagnato, "Infrared LED irradiation applied during high-intensity treadmill training improves maximal exercise tolerance in postmenopausal women: a 6-month longitudinal study", Lasers in Medical Science, (2013), pp. 1-8.

[40] A. E. Aquino Jr , M. Sene-Fiorese, F. R. Paolillo, F. O. Duarte, J. C. Oishi, A. A. Pena Jr, A. C. G. O. Duarte, M. R. Hamblin, V. S. Bagnato and N. A. Parizotto, "Low-level laser therapy (LLLT) combined with swimming training improved the lipid profile in rats fed with high-fat diet", Lasers Med Sci. (2012). 
International Journal of Advanced Science and Technology Vol.61, (2013) 